\title{
Elevada prevalência de obesidade abdominal em idosos e associação com diabetes, hipertensão e doenças respiratórias
}

\author{
High prevalence of abdominal obesity among the elderly and its \\ association with diabetes, hypertension and respiratory diseases
}

Erika Aparecida Silveira ${ }^{1}$

Liana Lima Vieira ${ }^{2}$

Jacqueline Danesio de Souza ${ }^{1}$

${ }^{1}$ Programa de PósGraduação em Ciências da Saúde, Faculdade de Medicina, Universidade Federal de Goiás (UFG). Primeira Avenida s/n, Setor Universitário. 74605 020 Goiânia GO Brasil. erikasil@terra.com.br

${ }^{2}$ Hospital das Clínicas, UFG.
Abstract Rapid population aging and abdominal obesity (AO) are worldwide public health problems among the elderly and have been insufficiently investigated. This article sets out to identify the prevalence of AO among the elderly, its association with different morbidities, sociodemographic variables, and lifestyle according to sex. It is a cross-sectional study with a representative sample of elderly people resident in Goiania, Brazil. Home visits were conducted to measure weight, height, waist circumference (WC), blood pressure, and to fill out a standardized form. The outcome variable, was calculated at WC> $102 \mathrm{~cm}$ for men and $>88 \mathrm{~cm}$ for women. Multiple Poisson regression was performed using a hierarchical model, adopting a significance level of 5\%. The prevalence of $A O$ was $55.1 \%$, with $65.5 \%$ in women and $34.8 \%$ in men, with a significant difference $(p<0.001)$. In men, AO was associated with age range between 70 and 74 years, the presence of respiratory diseases and high blood pressure. In women, AO continued to be associated with the presence of diabetes mellitus. The results contribute to the understanding of $A O$ and developing preventive approaches in public health.

Key words Abdominal obesity, Elderly, Diabetes mellitus, Hypertension, Respiratory diseases, Waist circumference
Resumo O envelhecimento populacional acelerado e a obesidade abdominal (OA) são problemas de saúde pública mundial entre idosos ainda pouco investigados. O artigo tem por objetivos identificar a prevalência da OA em idosos, verificar sua associação com diferentes morbidades e variáveis sociodemográficas e de estilo de vida, conforme sexo. Estudo transversal, com amostra representativa de idosos, residentes em Goiânia, Brasil. Foram realizadas visitas domiciliares para mensuração de peso, altura e circunferência abdominal (CA), pressão arterial e aplicação de questionário padronizado. A variável desfecho foi definida a partir da $C A \geq 102 \mathrm{~cm}$ nos homens $e \geq 88$ $\mathrm{cm}$ nas mulheres. Realizou-se regressão de Poisson múltipla, a partir de modelo hierárquico adotando-se nível de significância 5\%. A prevalência de OA foi de 55,1\%, sendo de 65,5\% nas mulheres e 34,8\% nos homens, com diferença significativa $(p<0,001)$. Nos homens a OA foi associada a faixa de idade entre 70 e 74 anos, presença de doenças do aparelho respiratório e hipertensão arterial. Nas mulheres, a OA manteve-se associada com a presença de diabetes mellitus. Os resultados contribuem para a compreensão da OA e o desenvolvimento de abordagens preventivas em saúde coletiva.

Palavras-chave Obesidade abdominal, Idoso, Diabetes mellitus, Hipertensão, Circunferência da cintura 


\section{Introdução}

O envelhecimento populacional é um fenômeno mundial que ocorre de forma acelerada ${ }^{1,2}$. No Brasil, a população idosa em 1970 correspondia a $4,95 \%$, percentual que chegou a $11 \%$, em $2010^{3}$. Com o aumento da expectativa de vida, observa-se também mudança no perfil de saúde, tornando-se predominante a ocorrência das doenças crônicas não transmissíveis $(\mathrm{DCNT})^{3}$. Esse cenário epidemiológico acarreta maiores custos aos serviços de saúde, devido o aumento das despesas com assistência médica e hospitalar, tanto no Sistema Único de Saúde (SUS) quanto na saúde suplementar ${ }^{4}$.

Dentro desse cenário epidemiológico, a obesidade abdominal (OA) é um forte fator de risco para diferentes DCNT ${ }^{5,6}$, com destaque para diabetes (DM) e doenças cardiovasculares (DC) ${ }^{7}$. Além disso, a OA está associada ao alto risco de mortalidade por algumas dessas doenças, independente do Índice de Massa Corporal (IMC) ${ }^{6,7}$. Atualmente, a circunferência abdominal (CA) destaca-se como o melhor preditor de gordura visceral quando comparado ao IMC em adultos ${ }^{7}$. Nos idosos, a OA relaciona-se com as alterações nas funções fisiológicas e metabólicas, que acabam por refletir na composição corporal e saúde da pessoa idosa ${ }^{8}$. Devido às mudanças relacionadas ao processo de envelhecimento, como o acúmulo de tecido adiposo 9 , torna-se fundamental identificar e avaliar o risco para o desenvolvimento ou a presença de DCNT e outras morbidades associadas a CA aumentada.

Alguns estudos internacionais ou desenvolvidos na região Sudeste e Sul do Brasil, avaliaram a prevalência de $\mathrm{OA}$ em idosos, principalmente nas idosas. Porém, investigações sobre associações, sexo específicas, e a presença concomitante de outras morbidades, principalmente as respiratórias, não foram localizadas na literatura cientifica, sendo esse, portanto, um aspecto importante a ser investigado ${ }^{10-13}$. Diante das grandes diversidades socioculturais e de saúde, existentes entre as regiões do país conhecer a prevalência de $\mathrm{OA}$ e os fatores associados em idosos da Região Centro-oeste do Brasil é um importante aspecto no campo da saúde pública, ainda mais considerando que não foram identificadas pesquisas com essa abordagem fora da Região Sudeste. Diante do exposto, os objetivos do estudo foram: identificar a prevalência da $\mathrm{OA}$ em idosos, verificar sua associação com diferentes morbidades de saúde e variáveis sociodemográficas e de estilo de vida, estratificando por sexo.

\section{Métodos}

Estudo transversal inserido no Projeto Idosos Goiânia ${ }^{10-13}$, com coleta de dados entre novembro de 2008 e março de 2009. Foram incluídos, idosos com 60 anos ou mais, de ambos os sexos, residentes em Goiânia-GO, não institucionalizados, usuários do SUS e que realizaram consultas nas unidades básicas de saúde nos 12 meses anteriores à pesquisa. Idosos com impossibilidade para a tomada das medidas antropométricas ou alguma incapacidade cognitiva ou auditiva para responder ao questionário não eram elegíveis. A participação dos idosos foi voluntária, mediante assinatura do Termo de Consentimento Livre Esclarecido, aprovado pelo do Comitê de Ética em Pesquisa da Universidade Federal de Goiás.

Realizou-se procedimento amostral probabilístico em múltiplos estágios de forma a representar a população idosa residente em cada um dos nove distritos sanitários do município de Goiânia-GO. Detalhamentos metodológicos sobre as etapas dos procedimentos amostrais do Projeto Idosos Goiânia já se encontram publicados previamente ${ }^{10-13}$.

O cálculo do tamanho amostral foi realizado a posteriori, com objetivo de verificar se o tamanho amostral do projeto matriz atendia ao presente estudo ${ }^{10-13}$. Para tanto considerou-se o desfecho OA, variável de exposição sexo feminino e os seguintes parâmetros: nível de confiança de $95 \%$, poder de $80 \%$, razão de não expostos: expostos de 1:1,9, razão de prevalência de 1,9, prevalência da doença entre os não expostos (homens) de 34,8\%. Com esse cálculo foi estimado 310 idosos, acrescentou $30 \%$ para estratificação e controle de fator de confusão, totalizando ao final 403 idosos. Assim, o número amostral do projeto matriz de 418 idosos foi suficiente para os objetivos desse estudo.

Durante as visitas domiciliares foi aplicado questionário estruturado, padronizado e prétestado em estudo piloto, tomado as medidas de peso, estatura e CA, além da aferição da pressão arterial. As medidas antropométricas (peso, CA e estatura) foram realizadas após padronização, para atingir precisão e acurácia inter e intra-antropometrista, com cálculos de erro técnico da medida $^{14,15}$. O peso foi medido em quilogramas, utilizando-se balança eletrônica digital portátil, devidamente calibrada, com capacidade de até $150 \mathrm{~kg}$ e precisão de $100 \mathrm{~g}$. A estatura foi aferida com a utilização de métrica de 2 metros e precisão de $0,1 \mathrm{~cm}$, fixada a uma parede lisa sem rodapé, com auxílio de fio de prumo e esquadro 
de madeira. A CA foi medida em centímetros, utilizando fita métrica inextensível e milimetrada com precisão de $1 \mathrm{~mm}$, posicionada no ponto médio entre a última costela e a crista ilíaca ${ }^{16}$. O peso, a CA e a estatura foram aferidos em duplicata, utilizando-se como resultado a média aritmética das medidas. O IMC foi calculado pela divisão do peso $(\mathrm{kg})$, pelo quadrado da estatura em metros $\left(\mathrm{kg} / \mathrm{m}^{2}\right)$ e classificado segundo critério proposto por Lipschitz ${ }^{17}$ e recomendado por Silveira et al. ${ }^{9}:$ IMC $<22 \mathrm{~kg} / \mathrm{m}^{2}$ para baixo peso, 22 a $27 \mathrm{~kg} / \mathrm{m}^{2}$ eutrofia e, a variável desfecho IMC $>27 \mathrm{~kg} / \mathrm{m}^{2}$.

As variáveis independentes analisadas foram: socioeconômicas e demográficas (sexo, idade, cor da pele, viver com companheiro, anos de estudo, classe econômica e paridade); estilo de vida (tabagismo, consumo de bebida alcoólica e sedentarismo) e presença de morbidades.

A classe econômica foi avaliada conforme o Critério de Classificação Econômica da Associação Brasileira de Empresas de Pesquisa - ABEP ${ }^{18}$. O nível de atividade física foi avaliado, categorizado e classificado segundo recomendações da Organização Panamericana de Saúde ${ }^{19}$. A presença ou ausência de sedentarismo foi verificada partir de quatro domínios: atividade física no lazer (inativo - ausência de atividade no lazer), atividade doméstica (inativo - nenhuma atividade doméstica pesada em menos de três dias na semana com duração inferior a três horas), atividade física no trabalho (inativo - ficar sentado a maior parte do tempo ou realizar apenas atividades de pouco esforço físico) e atividade física no deslocamento (inativo - deslocar de carro, moto, ônibus ou menos de dez minutos caminhando/ bicicleta). A partir disso, foi considerado sedentário o idoso inativo nos quatro domínios e não sedentário se o mesmo fosse ativo em pelo menos um dos domínios acima.

O consumo de bebida alcoólica foi verificado a partir do relato o tipo de bebida, a frequência e a quantidade (doses, garrafas, taças ou copos) consumida durante a semana anterior para, então, determinar a quantidade de gramas de etanol por dia de consumo dos idosos. Considerouse como fator de risco cardiovascular o consumo de bebida alcoólica com dosagem de etanol superior a $30 \mathrm{~g} /$ dia para homens e $15 \mathrm{~g} /$ dia para mulheres ${ }^{20,21}$.

As morbidades foram identificadas por meio das respostas à pergunta: "Quais doenças o seu médico já disse que o $\mathrm{Sr}$ (a) tem?”. Em seguida era lido uma lista de doenças as quais o idoso respondia sim ou não. Posteriormente foram cate- gorizadas conforme o número de morbidades, zero a um, dois a três e quatro ou mais. Nessa lista estavam incluídas as morbidades: infarto agudo do miocárdio, acidente vascular cerebral, diabetes, hipotireoidismo, doenças do sistema osteomuscular (artrite, artrose, osteoporose), doenças do aparelho digestório (gastrite, esofagite, diverticulite, úlcera gástrica, hérnia de hiato, colelitíase), doenças do aparelho respiratório (asma, doença pulmonar obstrutiva crônica, bronquite, enfisema pulmonar, sinusite), doenças infecciosas (chagas e hanseníase) e neoplasias (pele, mama, útero/ovário, próstata, cabeça e pescoço, pulmão, leucemia, outros locais) ${ }^{22}$. A presença de morbidades foi analisada e categorizada segundo a Classificação Estatística Internacional de Doenças e Problemas Relacionados à Saúde - CID1023.

A pressão arterial sistólica e diastólica (PAS e PAD) foi aferida com aparelho semi-automático (OMRON - HEM 705 CP) seguindo os critérios da VI Diretrizes Brasileiras de Hipertensão Arterial (2010). A PAS e PAD foram aferidas duas vezes utilizando-se como resultado a média aritmética das mesmas. Foram considerados hipertensos os idosos com PAS $\geq 140 \mathrm{mmHg}$ e/ou PAD $\geq 90 \mathrm{mmHg}$, ou em uso de medicação anti-hipertensiva $^{20}$.

Considerou-se em homens CA normal valor inferior a $94 \mathrm{~cm}$, aumentada entre 94 e $102 \mathrm{~cm}$ e muito aumentada maior ou igual a $102 \mathrm{~cm}$. Para mulheres, considerou-se CA normal valor inferior a $80 \mathrm{~cm}$, aumentada entre 80 e $88 \mathrm{~cm}$, e muito aumentada maior ou igual a $88 \mathrm{~cm}$. Denominou-se risco de OA para os valores de CA aumentada. Para a variável desfecho OA foi aplicado os valores de CA muito aumentada ${ }^{16,24}$.

$O$ banco de dados digitado em dupla entrada no EPI DATA ${ }^{\circledast}$ versão 3.1 para análise de consistência e validação. Já a análise estatística foi realizada no programa STATA/SE, versão 8.0. Foram calculadas a prevalência do desfecho para cada variável analisada, além de médias e frequências absolutas e relativas. Verificou-se a normalidade das variáveis contínuas e as médias foram comparadas pelo teste $t$ de Student.

Inicialmente, realizou-se análise bivariada pela regressão de Poisson simples, sendo a medida de efeito a razão de prevalência $(\mathrm{RP})$ e respectivo intervalo de confiança de 95\%, considerando-se nível de significância de 5\%. Considerando o desenho do estudo e o processo amostral, foi realizada análise por clusters, sendo estes os distritos sanitários. A partir de um modelo hierárquico de análise, foram incluídas na análise multivariada por regressão de Poisson as variáveis 
que apresentaram significância menor ou igual a 0,20 na análise bivariada, de modo que possíveis fatores de confusão da OA fossem controlados. No modelo de análise hierarquizado proposto, as variáveis socioeconômicas e demográficas constituíram o nível mais distal (nível 1), as variáveis estilo de vida, o nível intermediário (nível 2), e a presença de morbidades, o nível mais proximal (nível 3).

Iniciou-se o modelo com as variáveis do nível mais distal (nível 1) e a seguir foram introduzidos, um a um, os níveis subsequentes. Dentro de cada nível hierárquico foi considerado fator de risco para a $\mathrm{OA}$ as variáveis com $\mathrm{p}<0,05$, sendo que as mesmas permaneceram no modelo múltiplo nas análises dos níveis subsequentes. As variáveis que apresentaram valor de $\mathrm{p}$ acima de 0,05 foram retiradas do modelo multivariado. Todas as análises foram realizadas separadamente para sexo masculino e feminino.

\section{Resultados}

Foram avaliados 418 idosos, sendo $142(34,0 \%)$ homens e $276(66,0 \%)$ mulheres. Entre homens, a média da CA foi de $97,2 \mathrm{~cm} \pm 13,9$, nas mulheres foi de $91,6 \mathrm{~cm} \pm 11,9$, sendo estas médias estatisticamente diferentes $(\mathrm{p}<0,001)$. Não foi observada diferença estatística $(p=0,848)$ entre a média de IMC de homens $\left(30 \mathrm{~kg} / \mathrm{m}^{2} \pm 2,6\right)$ e mulheres $\left(30,1 \mathrm{~kg} / \mathrm{m}^{2} \pm 4,4\right)$ com OA.

A prevalência de OA na amostra foi de $55,1 \%$ $(\mathrm{n}=230)($ IC95\%: 49,9-59,6), sendo de 65,5\% (n $=150)($ IC95\%: 59,8-71,1) nas mulheres e 34,8\% $(\mathrm{n}=80)($ IC95\%: 26,8-42,7) nos homens $(\mathrm{p}<$ $0,001)$. O risco de OA apresentou prevalência de $21,2 \%(\mathrm{n}=89) \mathrm{na}$ amostra, $27,7 \%(\mathrm{n}=116)$ nos homens e $17,8 \%(\mathrm{n}=74)$ nas mulheres. Em mulheres, a prevalência de OA foi 1,9 vezes maior (IC95\%: 1,5-2,4) em relação aos homens.

Outras características da amostra estudada podem ser observadas na Tabela 1 , onde destacase no sexo masculino: maior percentual na faixa etária entre 65 a 74 anos $(54,9 \%)$, de cor da pele parda e preta $(55,6 \%)$, vivendo com companheira $(81,7 \%)$, que estudaram de 1 a 4 anos $(38,4 \%)$ e pertencentes a classe econômica C $(48,9 \%)$. Nas mulheres observou-se: cor da pele branca $(50,6 \%)$, maior frequência entre aquelas que vivem sem companheiro (59,1\%), estudou entre 1 e 4 anos (43,3\%), classe econômica C 46,9\%, 7 ou mais gravidezes na vida (45\%), sedentarismo em $62,2 \%$. Entre os homens e mulheres não se observou associação significativa entre OA e as variá- veis demográficas, socioeconômicas e de estilo de vida na análise bivariada (Tabela 1).

Para a presença de morbidades, verificou-se na análise bivariada, associação estatisticamente significante de OA com doenças do aparelho respiratório ( $\mathrm{RP}=1,89$ IC95\%:1,16-3,08) nos homens e com DM (RP = 1,31 IC95\%:1,11-1,54) nas mulheres (Tabela 2).

As variáveis incluídas no modelo de análise multivariada, para os homens, foram: idade, tabagismo, número de morbidades, doenças do aparelho respiratório e HAS. Em mulheres foram: cor da pele, viver com companheiro, paridade, acidente vascular cerebral (AVC), DM, hipotireoidismo, doenças do sistema osteomuscular, doenças respiratórias e HAS.

Após ajustes para potenciais fatores de confusão na análise multivariada, observou-se associação estatisticamente significante nos homens com OA na faixa de idade entre 70 e 74 anos (RP $=2,00$, IC95\%: 1,04-3,86), portadores de doenças do aparelho respiratório ( $\mathrm{RP}=2,32$, IC95\%: 1,28-4,17) e HAS ( RP = 1,93, IC95\%: 1,08-3,45). Nas mulheres, à OA manteve-se associada com a presença de DM (RP = 1,32, IC95\%: 1,12-1,56) (Tabela 3).

\section{Discussão}

O estudo identificou elevada prevalência de OA em idosos, acometendo mais de $50 \%$, além de ter investigado os fatores associados a OA tanto para os idosos do sexo masculino, quanto do sexo feminino. Outro aspecto de destaque da presente pesquisa foi a análise de um conjunto de doenças concomitantes a OA nos idosos. Essas são contribuições importantes para a compreensão do problema de obesidade abdominal em indivíduos idosos e que sai do eixo Sul-Sudeste, onde se concentram as pesquisas em idosos no Brasil.

A elevada prevalência de OA em idosos corrobora com estudos desenvolvidos nos estados do Paraná ${ }^{25}$ e Espírito Santo ${ }^{26}$, assim como na Espanha ${ }^{27}$. Verificou-se que o valor médio da CA foi diferente entre os gêneros, o que se assemelha com resultados de estudos nacionais ${ }^{25,26} \mathrm{e}$ internacionais ${ }^{27,28}$. Destaca-se que essa é a primeira pesquisa a analisar obesidade abdominal em idosos residentes na Região Centro-oeste do Brasil, assim contribui com informações sobre o problema da OA em idosos com perfil socioeconômico e epidemiológico difere das regiões sul e sudeste, onde as pesquisas populacionais com idosos foram realizadas. Devido à alta prevalência de $\mathrm{OA}$ 
Tabela 1. Prevalência de obesidade abdominal em homens e mulheres idosos segundo variáveis socioeconômicas, demográficas e estilo de vida. Projeto Idosos Goiânia, Brasil $(n=418)$.

\begin{tabular}{|c|c|c|c|c|c|c|}
\hline \multirow[b]{2}{*}{ Variáveis } & \multicolumn{3}{|c|}{ Homens } & \multicolumn{3}{|c|}{ Mulheres } \\
\hline & $\begin{array}{c}\text { Prevalência } \\
\text { n (\%) }\end{array}$ & $\begin{array}{c}\text { RP } \\
\text { (IC95\%) }\end{array}$ & Valor $\mathrm{p}^{*}$ & $\begin{array}{c}\text { Prevalência } \\
\text { n (\%) }\end{array}$ & RP (IC95\%) & Valor $\mathrm{p}^{*}$ \\
\hline Idade & & & 0,143 & & & $0,320^{\dagger}$ \\
\hline 60 a 64 & $9(33,33)$ & $1,37(0,63-2,99)$ & & $42(71,19)$ & $1,19(0,93-1,52)$ & \\
\hline 65 a 69 & $12(31,58)$ & $1,30(0,62-2,72)$ & & $48(62,34)$ & $1,04(0,81-1,34)$ & \\
\hline 70 a 74 & $19(48,72)$ & $2,00(1,04-3,86)$ & & $44(70,97)$ & $1,19(0,93-1,51)$ & \\
\hline 75 ou mais & $9(24,32)$ & 1,00 & & $46(59,74)$ & 1,00 & \\
\hline Cor da pele & & & 0,872 & & & 0,133 \\
\hline Branca & $22(35,48)$ & $1,04(0,66-1,64)$ & & $91(70,00)$ & $1,14(0,96-1,35)$ & \\
\hline Parda e preta & $27(34,18)$ & 1,00 & & $89(61,38)$ & 1,00 & \\
\hline Viver com companh & $o(a)$ & & 0,654 & & & 0,064 \\
\hline Sim & $39(33,91)$ & 1,00 & & $81(71,68)$ & $1,17(0,99-1,39)$ & \\
\hline Não & $10(38,46)$ & $1,13(0,65-1,97)$ & & $99(61,11)$ & 1,00 & \\
\hline Anos de estudo & & & $0,220^{\dagger}$ & & & 0,813 \\
\hline Analfabeto & $14(45,16)$ & $1,72(0,73-4,01)$ & & $52(64,20)$ & $1,14(0,72-1,81)$ & \\
\hline 1 a 4 & $16(32,00)$ & $1,21(0,51-2,86)$ & & $68(66,02)$ & $1,17(0,74-1,85)$ & \\
\hline 5 a 8 & $11(34,38)$ & $1,31(0,53-3,20)$ & & $28(70,00)$ & $1,24(0,77-2,00)$ & \\
\hline 9 ou mais & $5(26,32)$ & 1,00 & & $9(56,25)$ & 1,00 & \\
\hline Classe econômica & & & 0,572 & & & $0,590^{\dagger}$ \\
\hline $\mathrm{A} / \mathrm{B}$ & $12(42,86)$ & $1,34(0,77-2,33)$ & & $22(66,67)$ & $1,06(0,80-1,40)$ & \\
\hline $\mathrm{C}$ & $22(31,88)$ & 1,00 & & $82(66,13)$ & $1,05(0,87-1,27)$ & \\
\hline $\mathrm{D} / \mathrm{E}$ & $15(34,88)$ & $1,09(0,64-1,87)$ & & $71(62,83)$ & 1,00 & \\
\hline Paridade & & & & & & $0,060^{\dagger}$ \\
\hline zero & & & & $11(52,38)$ & 1,00 & \\
\hline 1 a 3 & & & & $33(60,00)$ & $1,14(0,72-1,82)$ & \\
\hline 4 a 6 & & & & $55(67,07)$ & $1,28(0,82-1,98)$ & \\
\hline 7 ou mais & & & & $81(70,43)$ & $1,34(0,88-2,05)$ & \\
\hline Tabagismo & & & $0,154^{\prime \prime}$ & & & $0,260^{\dagger}$ \\
\hline Fumante & $3(18,75)$ & 1,00 & & $94(62,67)$ & 1,00 & \\
\hline Ex-fumante & $32(41,56)$ & $2,21(0,77-6,38)$ & & $15(65,22)$ & $1,04(0,75-1,44)$ & \\
\hline Não fumante & $14(29,17)$ & $1,55(0,51-4,74)$ & & $71(69,61)$ & $1,11(0,93-1,33)$ & \\
\hline $\begin{array}{l}\text { Consumo bebida } \\
\text { alcoólica }\end{array}$ & & & 0,394 & & & 0,360 \\
\hline Não & $39(36,79)$ & $1,29(0,72-2,30)$ & & $159(64,63)$ & 1,00 & \\
\hline Sim & $10(28,57)$ & 1,00 & & $21(72,41)$ & $1,12(0,88-1,43)$ & \\
\hline Sedentarismo & & & 0,271 & & & 0,240 \\
\hline Sim & $25(39,68)$ & $1,29(0,82-2,03)$ & & $112(68,29)$ & $1,11(0,93-1,34)$ & \\
\hline Não & $24(30,77)$ & 1,00 & & $68(61,26)$ & 1,00 & \\
\hline
\end{tabular}

é importante avaliar esse problema em diferentes contextos socioeconômicos e culturais, principalmente no Brasil, devido a heterogeneidade das diferentes regiões ${ }^{25-28}$. As informações dessa pesquisa podem ser comparadas a idosos de outras regiões menos desenvolvidas na América
Latina e Ásia, além de possibilitar a comparação entre $\operatorname{sexos}^{25,29}$. Ressalta-se que a concentração de idosos dessa amostra foi em classes econômicas e escolaridade mais baixas.

A prevalência de OA foi maior nas mulheres (65,5\%), complicações relacionadas a este fato 
Tabela 2. Prevalência da obesidade abdominal em homens e mulheres idosos segundo presença de morbidades. Projeto Idosos Goiânia, Brasil ( $\mathrm{n}=418)$.

\begin{tabular}{|c|c|c|c|c|c|c|}
\hline \multirow[b]{2}{*}{ Variáveis } & \multicolumn{3}{|c|}{ Homens } & \multicolumn{3}{|c|}{ Mulheres } \\
\hline & $\begin{array}{c}\text { Prevalência } \\
\text { n (\%) }\end{array}$ & $\begin{array}{c}\text { RP } \\
\text { (IC95\%) }\end{array}$ & Valor $\mathbf{p}^{*}$ & $\begin{array}{c}\text { Prevalência } \\
\text { n (\%) }\end{array}$ & $\begin{array}{c}\text { RP } \\
\text { (IC95\%) }\end{array}$ & Valor $\mathbf{p}^{\star}$ \\
\hline $\mathrm{N}^{\circ}$ de morbidades & & & $0,120^{\dagger}$ & & & $0,270^{\dagger}$ \\
\hline 0 a 1 & $13(27,08)$ & 1,00 & & $40(63,49)$ & $1,01(0,80-1,26)$ & \\
\hline 2 a 3 & $26(36,62)$ & $1,35(0,77-2,36)$ & & $90(62,94)$ & 1,00 & \\
\hline 4 ou mais & $10(45,45)$ & $1,68(0,87-3,22)$ & & $50(72,46)$ & $1,15(0,95-1,39)$ & \\
\hline IAM & & & $0,576^{\ddagger}$ & & & $0,655^{\ddagger}$ \\
\hline Não & $46(34,85)$ & 1,00 & & $179(63,33)$ & 1,00 & \\
\hline Sim & $3(37,5)$ & $1,08(0,42-2,72)$ & & $1(100,00)$ & $1,53(1,40-1,67)$ & \\
\hline $\mathrm{AVC}$ & & & $0,531^{*}$ & & & $0,098^{*}$ \\
\hline Não & $47(35,34)$ & $1,24(0,37-4,10)$ & & $177(66,29)$ & $1,77(0,72-4,35)$ & \\
\hline Sim & $2(28,57)$ & 1,00 & & $3(37,50)$ & 1,00 & \\
\hline Diabetes & & & 0,538 & & & 0,001 \\
\hline Não & $36(33,64)$ & 1,00 & & $128(60,95)$ & 1,00 & \\
\hline Sim & $13(39,39)$ & $1,17(0,71-1,93)$ & & $52(80,00)$ & $1,31(1,11-1,54)$ & \\
\hline Hipotireoidismo & & & & & & 0,146 \\
\hline Não & & & & $163(64,43)$ & 1,00 & \\
\hline Sim & & & & $17(77,27)$ & $1,20(0,94-1,53)$ & \\
\hline \multicolumn{2}{|c|}{ Doenças do Sistema Osteomuscular } & & 0,779 & & & 0,094 \\
\hline Não & $39(34,21)$ & 1,00 & & $99(61,49)$ & 1,00 & \\
\hline Sim & $10(37,04)$ & $1,08(0,62-1,89)$ & & $81(71,05)$ & $1,16(0,97-1,37$ & \\
\hline \multicolumn{2}{|c|}{ Doenças do Aparelho Digestivo } & & 0,960 & & & 0,982 \\
\hline Não & $43(34,68)$ & 1,00 & & $159(65,43)$ & 1,00 & \\
\hline Sim & $6(35,29)$ & $1,02(0,51-2,03)$ & & $21(65,63)$ & $1,00(0,77-1,31)$ & \\
\hline \multicolumn{2}{|c|}{ Doenças do Aparelho Respiratório } & & 0,010 & & & 0,161 \\
\hline Não & $40(31,75)$ & 1,00 & & $165(67,07)$ & $1,30(0,901,86)$ & \\
\hline $\operatorname{Sim}$ & $9(60,00)$ & $1,89(1,16-3,08)$ & & $15(51,72)$ & 1,00 & \\
\hline \multicolumn{2}{|l|}{ Doenças Infecciosas } & & $0,694^{\ddagger}$ & & & 0,586 \\
\hline Não & $46(34,33)$ & 1,00 & & $170(65,89)$ & $1,12(0,74-1,68)$ & \\
\hline Sim & $3(42,86)$ & $1,25(0,51-3,04)$ & & $10(58,82)$ & 1,00 & \\
\hline \multicolumn{2}{|c|}{ Doenças Neoplásicas } & & $0,342^{\ddagger}$ & & & $0,419^{*}$ \\
\hline Não & $46(33,82)$ & 1,00 & & $177(65,80)$ & $1,32(0,59-2,95)$ & \\
\hline Sim & $3(60,00)$ & $1,77(0,83-3,78)$ & & $3(50,00)$ & 1,00 & \\
\hline \multicolumn{2}{|c|}{ Hipertensão arterial aferida } & & 0,177 & & & 0,063 \\
\hline Não & $8(24,24)$ & 1,00 & & $25(52,08)$ & 1,00 & \\
\hline Sim & $41(37,96)$ & $1,56(0,81-3,00)$ & & $155(68,28)$ & $1,31(0,98-1,74)$ & \\
\hline
\end{tabular}

* Teste de Wald ${ }^{\dagger}$ Qui-quadrado de tendência linear ${ }^{\ddagger}$ Teste exato de Fisher IAM = Infarto agudo do miocárdio AVC = Acidente vascular cerebral D = doenças segundo Classificação Estatística Internacional de Doenças e Problemas Relacionados à Saúde CID-10.

são demonstradas em estudos populacionais e transversais ${ }^{25,26,30,31}$. Fatores relacionados ao aumento da mortalidade podem explicar esses achados: as mulheres acumulam mais gordura visceral e subcutânea do que os homens; há diferenças no padrão alimentar entre os sexos; a menopausa é acompanhada por aumento de peso e adiposidade $\mathrm{e}^{7,32,33}$
A prevalência de obesidade aumentou até os 74 anos nos homens, com decréscimo a partir dos 75 anos. Esse achado poderia ser interpretado como um possível viés de sobrevivência, ou seja, a mortalidade daqueles com OA a partir dos 75 anos. Nesse caso, a OA levaria a uma maior carga de DCNT em grupos etários mais jovens, que não sobreviveriam até idades mais avança- 
Tabela 3. Modelo final de análise multivariada de obesidade abdominal em homens e mulheres idosos. Projeto Idosos Goiânia, Brasil ( $\mathrm{n}=418)$.

\begin{tabular}{|c|c|c|c|c|}
\hline \multirow{2}{*}{ Variáveis } & RP ajustada & IC95\% ajustada & Valor $\mathbf{p}^{*}$ & Valor $\mathrm{p}^{\star}$ \\
\hline & \multicolumn{4}{|c|}{ Homens } \\
\hline Idade & & & & 0,143 \\
\hline 60 a 64 & 1,37 & $0,63-3,00$ & 0,430 & \\
\hline 65 a 69 & 1,30 & $0,62-2,72$ & 0,489 & \\
\hline 70 a 74 & 2,00 & $1,04-3,86$ & 0,038 & \\
\hline 75 ou mais & 1,00 & & & \\
\hline Doenças do Aparelho Respiratório & & & & 0,005 \\
\hline Não & 1,00 & & & \\
\hline Sim & 2,32 & $1,28-4,17$ & 0,005 & \\
\hline Hipertensão arterial & & & & 0,025 \\
\hline Não & 1,00 & & & \\
\hline \multirow[t]{2}{*}{ Sim } & 1,93 & $1,08-3,45$ & 0,025 & \\
\hline & \multicolumn{4}{|c|}{ Mulheres } \\
\hline Diabetes & & & & 0,001 \\
\hline Não & 1,00 & & & \\
\hline Sim & 1,32 & $1,12-1,56$ & 0,001 & \\
\hline
\end{tabular}

* Teste de Wald.

das. Os resultados deste estudo confirmam o já estabelecido em outros estudos com idosos, mostrando que o excesso de peso, e não a desnutrição, constitui o principal problema nutricional da população idosa brasileira ${ }^{25,26}$.

A OA foi associada com a presença de HAS em homens, resultado confirmado por estudo com a população europeia, onde é estabelecida associação positiva entre OA com $\mathrm{HAS}^{27}$. Já em estudo envolvendo mulheres idosas, a medida da circunferência da cintura é considera como forte preditor de $\mathrm{HAS}^{25}$. Estudos com idosos de ambos os sexos estabelece aumento do risco de doenças cardiovasculares relacionados à distribuição de gordura em determinadas regiões, com destaque para deposição de gordura na região abdominal $^{30-32}$.

A associação de DM com OA em mulheres idosas observada nessa pesquisa confirma achados de outros estudos com idosas $27,30,31$. O aumento da gordura visceral está relacionado com a resistência insulínica e elevado risco de DM tipo 2, uma vez que o tecido adiposo visceral secreta adiponectina e outras substâncias vasoativas importante na regulação do metabolismo da glicose, contribuindo para o desenvolvimento do DM tipo $2^{5,31}$.

Nos homens a OA foi associada também à presença de doenças respiratórias. Esse é um resultado importante no estudo da saúde de idosos, uma vez que sua compreensão pode se basear em estudo realizado em adultos que verificou a $\mathrm{OA}$ como fator associado ao comprometimento da função pulmonar e padrão ventilatório restritivo, independente do tabagismo e $\mathrm{IMC}^{34}$. Essa associação pode ser resultado tanto de efeitos mecânicos quanto de efeitos metabólicos do tecido $\operatorname{adiposo}^{35}$.

Com relação as limitações do estudo, vale lembrar que o delineamento transversal impossibilita fazer inferências causais devido a falta de temporalidade para verificar o efeito ao longo do tempo da OA sobre doenças crônicas como HAS, DM e doenças respiratórias nos idosos. Os pontos de CA até então recomendados e aplicados na presente pesquisa, possuem validação para população adulta, porém estudos têm discutido as limitações inerentes aos mesmos quando aplicados na população de idosos ${ }^{36,37}$. Quanto aos aspectos metodológicos a pesquisa tomou diversos cuidados para garantir precisão e acurácia das medidas antropométricas e também na seleção da amostra, coleta de dados, estruturação do banco de dados e análises estatísticas.

\section{Conclusão}

Os achados da presente pesquisa demonstram elevada prevalência de OA em idosos de ambos 
os sexos, com destaque para as mulheres, além da associação com a presença de outras doenças como DM, HAS e doenças respiratórias. É relevante a associação entre OA e as demais morbidades, ainda mais considerando que estes são fatores de risco potências para o desenvolvimento de doenças cardiovasculares. Dessa forma, verificar a presença concomitante desses relevantes problemas de saúde nos idosos é um achado preocupante que necessita de reflexões sobre abordagens preventivas em saúde coletiva, visando estabelecer medidas para adequado manejo clínico da OA por profissionais de saúde.

\section{Colaboradores}

EA Silveira, LL Vieira e JD Souza participaram da autoria do manuscrito, nos seguintes termos: contribuição substancial para a concepção e planejamento; análise e interpretação dos dados; contribuição significativa na elaboração do rascunho ou na revisão crítica do conteúdo; participação da aprovação da versão final do manuscrito. A autora EA Silveira foi a coordenadora do Projeto em que este estudo está inserido, conhecido como Projeto Idosos Goiânia. 


\section{Referências}

1. Vidal PM, Bochud M, Mooser V, Paccaud F, Waeber G, Vollenweider P. Prevalence of obesity and abdominal obesity in the Lausanne population. BMC Public Health 2008; 8:330.

2. World Health Organization (WHO). Obesity: preventing and managing the global epidemic: report of a WHO consultation. Geneva: WHO; 1999.

3. Instituto Brasileiro de Geografia e Estatística (IBGE). Séries estatísticas \& séries históricas. Rio de Janeiro: IBGE; 2010

4. Lourenço RA, Martins CSF, Sanchez MA, Veras RP. Assistência ambulatorial geriátrica: hierarquização da demanda. Rev Saude Publica 2005; 39(2):311-318.

5. Yamamoto S, Nakagawa T, Matsushita Y, Kusano S, Hayashi T, Irokawa M, Aoki T, Korogi Y, Mizoue T. Fat Area and Markers of Insulin Resistance in Relation to Colorectal Neoplasia. Diabetes Care 2010; 33(1):184189.

6. Jacobs EJ, Newton CC, Wan Y, Patel AV, McCullough ML, Campbell PT, Aoki T, Korogi Y, Mizoue T. Waist Circumference and All-Cause Mortality in a Large US Cohort. Arch Intern Med 2010; 170(15):1293-1301.

7. Canoy D, Cairns BJ, Balkwill A, Wright FL, Green J, Reeves G, Beral V; Million Women Study Collaborators. Coronary heart disease incidence in women by waist circumference within categories of body mass index. Eur J Prev Cardiol 2013; 20(5):759-762.

8. Roopakala MS, Suresh A, Ashtalakshmi, Srinath, Ashok, Giridhar, Anand, Silvia WD. Anthropometric measurements as predictors of intraabdominal fat thickness. Indian J Physiol Pharmacol 2009; 53(3):259-264.

9. Silveira EA, Kac G, Barbosa LS. Prevalência e fatores associados à obesidade em idosos residentes em Pelotas, Rio Grande do Sul, Brasil: classificação da obesidade segundo dois pontos de corte do índice de massa corporal. Cad Saúde Pública 2009; 25(7):1569-1577.

10. Ferreira CCC, Peixoto MRG, Barbosa MA, Silveira EA. Prevalência de Fatores de Risco Cardiovascular em Idosos Usuários do Sistema Único de Saúde de Goiânia. Arq Bras Cardiol 2010; 95(5):621-628.

11. Pagotto V, Nakatani AYE, Silveira EA. Fatores associados à autoavaliação de saúde ruim em idosos usuários do Sistema Único de Saúde. Cad Saúde Pública 2011; 27(8):1593-1602.

12. Pagotto V, Silveira EA, Velasco WD. Perfil das hospitalizações e fatores associados em idosos usuários do SUS. Cien Saude Colet 2013; 18(10):3061-3070.

13. Pagotto V, Silveira EA. Applicability and agreement of different diagnostic criteria for sarcopenia estimation in the elderly. Arch Gerontol Geriatr 2014; 59(2):288294

14. Lohman TG, Roche AF, Martorel R. Anthropometric standardization reference manual. Illinois: Human Kinetics Books; 1988.

15. Habicht JP. Estandarizacion de métodos epidemiológicos cuantitativos sobre el terreno. Boletín de la Oficina Sanitaria Panamericana 1974; 76(5):375-381.

16. Han TS, Van Leer Em, Seidell JC, Lean MEJ. Waist circumference action levels in the identification of cardiovascular risk factors: prevalence study in a random sample. BMJ 1995; 311 (7017):1401-1405.

17. Lipschitz DA. Screening for nutritional status in the elderly. Prim Care 1994; 21(1):55-67.
18. Associação Brasileira de Empresas de Pesquisas [homepage na Internet]. Critério de Classificação Econômica Brasil. 2008 [citado 2009 Set 12]. Disponível em: www. abep.org/codigosguias/CCEB2008-Base2006e2007.pdf

19. Organización Panamericana de la Salud (OPAS). Protocolo y directrices: Conjunto de Ações para la Reducción Multifactorial de Enfermedades no Transmisibles (CARMEN/CINDI). Washington: OPAS; 1997.

20. Sociedade Brasileira de Cardiologia, Sociedade Brasileira de Hipertensão, Sociedade Brasileira de Nefrologia. VI Diretrizes Brasileiras para Hipertensão. Arq Bras Cardiol 2010; 95(1 Supl. 1):1-51.

21. Franco G. Tabela de Composição Química dos Alimentos. 9a ed. Rio de Janeiro: Atheneu; 1999.

22. Schienkiewitz A, Mensink GBM, Scheidt-Nave C. Comorbidity of overweight and obesity in a nationally representative sample of German adults aged 18-79 years. BMC Public Health 2012; 12:658.

23. Organização Mundial de Saúde (OMS). Classificação Estatística Internacional de Doenças e Problemas Relacionados à Saúde - CID-10. Centro Colaborador da OMS para a Classificação de Doenças em Português, décima revisão. São Paulo: Editora da Universidade de São Paulo; 2008 .

24. World Health Organization (WHO). Obesity: preventing and managing the global epidemic. Geneva: WHO; 1997.

25. Girotto E, Andrade SMD, Cabrera MAS. Prevalência de obesidade abdominal em hipertensos cadastrados em uma Unidade de Saúde da Família. Arq Bras Cardiol 2010; 94(6):754-762.

26. Andrade FB, Caldas Junior AF, Kitoko PM, Batista JE, Andrade TB. Prevalence of overweight and obesity in elderly people from Vitória-ES, Brazil. Cien Saude Colet 2012; 17(3):749-756.

27. Félix-Redondo FJ, Grau M, Baena-Díez JM, Dégano IR, de León AC, Guembe MJ, Alzamora MT, Vega-Alonso T, Robles NR, Ortiz H, Rigo F, Mayoral-Sanchez E, Tormo MJ, Segura-Fragoso A, Fernández-Bergés D. Prevalence of obesity and associated cardiovascular risk: the DARIOS study. BMC Public Health 2013; 13:542.

28. Zhuo Q, Wang Zhi-Qing, Fu P, Pião Jian-Hua, Tian Y, Xu J, Yang XG. Association between Adiponectin and Metabolic Syndrome in Older Adults from Major Cities of China. Biomed Environ Sci 2010; 23:53-61.

29. Yin Z, Shi X, Kraus VB, Brasher MS, Chen H, Liu Y, Lv Y, Zeng Y. Gender-dependent association of body mass index and waist circumference with disability in the Chinese oldest old. Obesity (Silver Spring) 2014; 22(8):1918-1925.

30. Schienkiewitz A, Mensink GBM, Scheidt-Nav C. Comorbidity of overweight and obesity in a nationally representative sample of German adults aged 18-79 years. BMC Public Health 2012, 12:658.

31. Taylor AE, Ebrahim S, Ben-Shlomo Y, Martin RM, Whincup PH, Yarnell JW, Wannamethee SG, Lawlor DA. Comparison of the associations of body mass index and measures of central adiposity and fat mass with coronary heart disease, diabetes, and all-cause mortality: a study using data from 4 UK cohorts. Am J Clin Nutr 2010; 91(3):547-556. 
32. Canoy D, Cairns BJ, Balkwill A, Wright FL, Green J, Reeves G, Beral V; Million Women Study Collaborators. Coronary heart disease incidence in women by waist circumference within categories of body mass index. Eur J Prev Cardiol 2013; 20(5):759-762.

33. Woodrow G. Body composition analysis techniques in the aged adult: indications and limitations. Curr Opin Clin Nutr Metab Care 2009; 12(1):8-14

34. ILeone N, Courbon D, Thomas F, Bean K, Jégo B, Leynaert B, Guize L, Zureik M. Lung function impairment and metabolic syndrome: the critical role of abdominal obesity. Am J Respir Crit Care Med 2009; 179(6):509516.

35. McClean KM, Kee F, Young IS, Elborn JS. Obesity and the lung: 1. Epidemiology. Thorax 2008; 63(7):649-654.

36. Florath I, Brandt S, Weck M N, Moss A, Gottmann P, Rothenbacher D, Wabitsch M, Brenner H. Evidence of inappropriate cardiovascular risk assessment in middle-age women based on recommended cutpoints for waist circumference. Nutr Metab Cardiovasc Dis 2014; 24(10):1112-1119.

37. Oliveira A, Cocate PG, Hermsdorff HH, Bressan J, Silva MF, Rodrigues JA, Natali AJ. Waist circumference measures: cutoff analyses to detect obesity and cardiometabolic risk factors in a Southeast Brazilian middle-aged men population--a cross-sectional study. Lipids Health Dis. 2014; 13:141.

Artigo apresentado em 12/09/2015

Aprovado em 25/05/2016

Versão final apresentada em 27/05/2016 